\title{
ELLIPTIC SOLITONS AND GRÖBNER BASES
}

\author{
YURII V.BREZHNEV
}

\begin{abstract}
We consider the solution of spectral problems with elliptic coefficients in the framework of the Hermite ansatz. We show that the search for exactly solvable potentials and their spectral characteristics is reduced to a system of polynomial equations solvable by the Gröbner bases method and others. New integrable potentials and corresponding solutions of the Sawada-Kotera, Kaup-Kupershmidt, Boussinesq equations and others are found.
\end{abstract}

\section{INTRODUCTION}

The paper is devoted to the algorithmic problems associated with integrating the spectral problems

$$
\widehat{L} \Psi \equiv \frac{d^{n}}{d x^{n}} \Psi(x ; \lambda)+u_{1}(x, \lambda) \frac{d^{n-1}}{d x^{n-1}} \Psi(x ; \lambda)+\cdots+u_{n}(x, \lambda) \Psi(x ; \lambda)=0,
$$

where $u_{j}$ are elliptic functions of $x$ and arbitrary (rational or transcendental) functions of $\lambda$. We shall restrict our consideration to the Schrödinger equation

$$
\Psi^{\prime \prime}-u(x) \Psi=\lambda \Psi,
$$

the equation

$$
\Psi^{\prime \prime \prime}-u(x) \Psi^{\prime}=\lambda \Psi
$$

and the generalization of the Halphen equation

$$
\Psi^{\prime \prime \prime}-u(x) \Psi^{\prime}-v(x) \Psi=\lambda \Psi .
$$

We use the term potential for the $u(x), v(x)$-functions. Until the 1970/80's, few exactly solvable potentials were known. Earlier, in 1872, Hermite [27] developed an approach for the integration of the Lamé equation

$$
\Psi^{\prime \prime}-n(n+1) \wp(x) \Psi=\lambda \Psi,
$$

and later, Halphen extended it to the third order equation

$$
\Psi^{\prime \prime \prime}-\left(n^{2}-1\right) \wp(x) \Psi^{\prime}-\frac{1}{2}\left(n^{2}-1\right) \wp^{\prime}(x) \Psi=\lambda \Psi .
$$

Here and below $\sigma, \zeta, \wp, \wp^{\prime}$ denote the standard Weierstrassian functions. See 24 , 18, 28 for an extensive discussion of these classical examples. According to modern terminology, the set of exactly solvable elliptic potentials is a particular case

Date: 1 April 2002.

1991 Mathematics Subject Classification. Primary 58F07; Secondary 34L05, 35Q53.

Key words and phrases. Elliptic solitons, finite-gap integration, spectral problems, integrable PDE's. 
of finite-gap potentials in elliptic functions. An intense investigation of elliptic potentials was initiated by the paper [29], and in 1987 Verdier and Treibich 440, 37] unexpectedly found new potentials for the equation (2) in elliptic functions

$$
u(x)=6 \wp(x)+2 \wp\left(x-\omega_{j}\right)
$$

and gave the term elliptic solitons to them. Recently V. Matveev drew attention to the fact that such potentials, in Jacobian form, were already considered by Darboux in a short note [11] in 1882. The following year, two comprehensive mémoires by Sparre [35] appeared on this topic.

The development of a theory led to the current result that elliptic solitons are the widest class of finite-gap explicit solutions. See, for example, [33, 16], recent results in [34, 36] and references therein. The monograph [1] reviews work in finite-gap theory up to the beginning of the 1990's and the review 21] and preparing book 23 contain a wide bibliography on that score.

One feature of elliptic solitons is the potential, and the $\Psi$-function can be found by the Hermite ansatz method [27. In the case of the potentials with the only pole in a parallelogram of periods, the derivation of the algebraic curve and other characteristics is not difficult. For this purpose it is enough to take a few resultants 14. 1, but in the general case the elimination technique is insufficient. Sect. 2 contains a pure algebraic interpretation of Hermite's method. In Sect. 3, we show that the general scheme for solving the problem under consideration (1) is reduced to the computation of the Gröbner basis for some polynomial system. After Buchberger's discovery in 1960's of an algorithm for finding the polynomial ideal bases, this area of algorithmic mathematics has rapidly developed. See [31] with regard to the modern achievements in this area. Sect. 4 contains a relation between the method and traditional objects in finite-gap integration theory: the canonical form of an algebraic curve $\widetilde{F}(\mu, \lambda)=0$ and reduction of one of the holomorphic differentials to the elliptic. Some new examples of elliptic solitons and their applications to the integrable partial differential equations (PDE's) are presented in Sects. 5-6 and development of the theory is discussed in Sect. 7.

\section{Algebraic characterization of the Hermite method}

Based on the $\Phi$-function

$$
\Phi(x ; \alpha)=\frac{\sigma(\alpha-x)}{\sigma(\alpha) \sigma(x)} e^{\zeta(\alpha) x}
$$

or more precisely l'élément simple

$$
\Phi(x ; \alpha, k)=\frac{\sigma(\alpha-x)}{\sigma(\alpha) \sigma(x)} e^{(\zeta(\alpha)+k) x}
$$

by Halphen [25, 26], Hermite and Halphen [27, 24] considered the following ansatz for the $\Psi$-function

$$
\Psi=\Phi(x ; \alpha, k)+a_{1} \Phi^{\prime}(x ; \alpha, k)+\cdots+a_{n} \Phi^{(n)}(x ; \alpha, k) .
$$

The function $\Phi(x ; \alpha, k)$ as well its $x$-derivatives $\Phi^{\prime}(x ; \alpha, k), \ldots, \Phi^{(n)}(x ; \alpha, k)$ are doubly-periodic functions of $x$ of the second kind. According to (1) and (8), the expression $\widehat{L} \Psi / \Phi\left(x-x_{0} ; \alpha, k\right)$ is a 2-periodic meromorphic function with only one simple pole at the point $x_{0}$. It must be a constant function. Setting it to be equal 
to zero, we have $\widehat{L} \Psi=0$ under the corresponding choice of additional parameters $k, \alpha$ and $a_{j}$. As the $\Phi(x ; \alpha, k)$-function has the first order pole at $x=0$ [25, p. 231]

$$
\Phi(x ; \alpha, k)=\frac{1}{x}+k+\frac{k^{2}-\wp(\alpha)}{2} x+\frac{k^{3}-3 \wp(\alpha) k+\wp^{\prime}(\alpha)}{6} x^{2}+\cdots
$$

to solve the problem, it is sufficient to equate to zero only the principal part(s) of the Laurent's expansion(s) of the expression $\widehat{L} \Psi$, where $\Psi$ is the ansatz (9) or its multi-pole generalizations (see Examples 1-3, $\mathbf{7}$ in Sect. 5).

As a nontrivial example we shall consider the 5-gap Lamé potential with $n=5$ in (5). It has been studied in [14, but we give a more simple solution. Note that the cases $n=2,3,4$ and partially 5 were considered already in [26, pp. 527-531]. In the same place one can find mention of eliminations.

By Frobenius theory, $\Psi$ must have a 5 -th order pole at $x=0$ and therefore the ansatz for the $\Psi$-function should be the following:

$$
\Psi=\Phi+a_{1} \Phi^{\prime}+\cdots+a_{4} \Phi^{(\mathrm{iv})} .
$$

Substituting (10) in (5) and expanding the result at $x=0$, we obtain a system of equations in the variables $k, \alpha, a_{j}$. This system is linear with respect to $a_{j}$. We do not write expressions for the $a_{j}$ ([14, formula (3.7)]). The remaining equations have the form

$$
\begin{aligned}
w_{1} \equiv & -6 k^{5}+\frac{20}{3}(9 \wp+\lambda) k^{3}-60 \wp^{\prime} k^{2}+\left(90 \wp^{2}-20 \lambda \wp-\frac{10}{7} \lambda^{2}+\frac{144}{7} g_{2}\right) k- \\
& -\frac{4}{3}(9 \wp-5 \lambda) \wp^{\prime}, \\
w_{2} \equiv & -5 k^{6}+(75 \wp+5 \lambda) k^{4}-100 \wp^{\prime} k^{3}+\left(225 \wp^{2}-30 \lambda \wp-\frac{5}{7} \lambda^{2}+\frac{180}{7} g_{2}\right) k^{2}- \\
& -20(3 \wp-\lambda) \wp^{\prime} k+25 \wp^{3}-15 \lambda \wp^{2}+\frac{5}{7}\left(\lambda^{2}-20 g_{2}\right) \wp-40 g_{3}-\frac{1}{21} \lambda^{3}+\frac{44}{7} g_{2} \lambda,
\end{aligned}
$$

which are understood to be equal to zero. The argument $\alpha$ in the $\wp, \wp^{\prime}$-functions is omitted for brevity. The system (2) has to be considered as algebraic with respect to $k$ and transcendental in $\alpha$. We emphasize that everywhere in the paper $\lambda$ is a parameter, but not variable in polynomial bases. Insomuch as functions $\wp(\alpha)$ and $\wp^{\prime}(\alpha)$ are related by the Weierstrass equation (torus)

$$
w_{3} \equiv \wp^{\prime}(\alpha)^{2}-4 \wp(\alpha)^{3}+g_{2} \wp(\alpha)+g_{3},
$$

we supplement (2) by (11) and consider (2 11) as a polynomial system with respect to independent variables $\left(k, \wp, \wp^{\prime}\right)$. The simplest method of solution consists of the elimination of the variable $\wp$ followed by $\wp^{\prime}$. As a result, we find that $k$ must be a root of the polynomial

$$
k^{4}\left(5103 k^{4}-945 \lambda k^{2}+40 \lambda^{2}+54 g_{2}\right)^{4}\left(225\left(27 g_{2}-\lambda^{2}\right) P_{6}^{2}(\lambda) k^{2}+P_{9}(\lambda) P_{3}^{2}(\lambda)\right),
$$

where $P_{6,9,3}(\lambda)$ are some polynomials in $\lambda$ with degrees $6,9,3$ respectively. It is not difficult to guess that the correct result requires that $k$ and $\lambda$ are related by the following equation

$$
F(k, \lambda): \quad 225\left(27 g_{2}-\lambda^{2}\right) P_{6}^{2}(\lambda) k^{2}+P_{9}(\lambda) P_{3}^{2}(\lambda)=0,
$$

since the differential equation (5) is of second order and we must have not more than 2 solutions for $k$ with fixed $\lambda$. Curve (12) can be brought into the canonical hyperelliptic form

$$
\widetilde{F}(\mu, \lambda): \quad \mu^{2}=\left(27 g_{2}-\lambda^{2}\right) P_{9}(\lambda)
$$


by an obvious birational transformation (note a misprint $27 g_{2}^{2}$ in this formula in 14. formula (3.8)]). The variables $\wp, \wp^{\prime}$ as functions of $k$ can be found in the same way: by the sequential reduction of exponents of $\wp, \wp^{\prime}$ in (2-11).

Obviously, the resultant technique is almost impossible if the potential has several poles [16, 33], as the number of variables increases. Another approach consists of finding an equivalent system with the following criterion. It is advisable for the new system to contain linear equations in $\wp, \wp^{\prime}$. These equations define $\alpha$ as a function of $(k, \lambda)$ :

$$
\wp(\alpha)=R_{1}(k ; \lambda), \quad \wp^{\prime}(\alpha)=R_{2}(k ; \lambda)
$$

and we call (13) a cover of torus (11) in algebraic form. Suppose one of the new equations does not contain $\wp, \wp^{\prime}$ (i.e. be an univariate polynomial in $k$ ) if the nontrivial solution for $k$ exists. We interpret such a polynomial as the algebraic curve $F(k, \lambda)=0$ corresponding to an elliptic potential. If $F(k, \lambda)$ has a factorised form then each of factors is investigated separately. The curve is one of them. It is clear that its degree in $k$ has to be equal to the order $n$ of the equation (1). The canonical form $\widetilde{F}(\mu, \lambda)=0$ of the curve is obtained with the help of a birational transformation between variables $(k, \lambda) \leftrightarrow(\mu, \lambda)$ (see an explanation in Sect. 4). Note, there are specialized algorithms for the computation of the univariate polynomial in an ideal without solving the system as a whole.

\section{Gröbner BASES, CURVES AND COVERS}

We clarify the main idea using the previous example. Let us consider three polynomials $w_{1,2,3}\left(\wp^{\prime}, \wp, k\right)$ as a system generating an ideal in a polynomial ring $\mathbb{Q}\left(\lambda, g_{2}, g_{3}\right)\left[\wp^{\prime}, \wp, k\right]$

$$
\langle I\rangle=h_{1} w_{1}+h_{2} w_{2}+h_{3} w_{3},
$$

where $h_{j}=h_{j}\left(\wp^{\prime}, \wp, k\right)$ are arbitrary elements of the ring. As is well known, the structure of the solution of the polynomial systems depends on the monomial ordering in a ring 31]. The arguments at the end of Sect. 2 (see also the elimination theorem in [10]) lead to the choice of pure lexicographic ordering $\wp^{\prime} \succ \wp \succ k$. The monograph [10] contains a good exposition of details of this subject. The main property of the Gröbner base is expressed in

Definition [10]. Let $\left\{w_{1}, w_{2}, \ldots\right\}$ be a basis of ideal $I=\left\langle w_{1}, w_{2}, \ldots\right\rangle$. Let $\succ$ be a monomial ordering on the ring $\mathbb{Q}[\ldots]$ and $\mathrm{LT}(f)$ denote the leading term (monomial) of a polynomial $f \in \mathbb{Q}[\ldots]$. The set $G=\left\{f_{1}, f_{2}, \ldots, f_{N}\right\}$ is said to be a standard basis (Gröbner Basis) if the monomial ideal generated by

$$
\left\langle\operatorname{LT}\left(f_{1}\right), \operatorname{LT}\left(f_{2}\right), \ldots, \operatorname{LT}\left(f_{N}\right)\right\rangle
$$

is coincident with an ideal $\langle\mathrm{LT}(I)\rangle$ generated by all the leading terms of $I$.

In other words, the leading term of any polynomial in $I$ is divisible by one of the $\mathrm{LT}\left(f_{j}\right)$. According to the definition at the end of Sect. 2 , the polynomial $F(k ; \lambda)$, determining the algebraic curve

$$
F(k ; \lambda)=0,
$$

is a generator of the intersection of the ideal $I$ and the ring of all polynomials in $k$ :

$$
\langle F(k ; \lambda)\rangle=I \cap \mathbb{Q}(\lambda)[k] .
$$

Thus we arrive at a general recipe for the solution of the spectral problem (1). 
Proposition. Let $\left\{w_{1}, w_{2}, \ldots\right\}$ be polynomials in $\wp^{\prime}(\alpha), \wp(\alpha), k, \ldots$ appearing in the Hermite method and determining the solution of the spectral problem (1): the cover of torus (13) and the curve (14). Then

1) Algorithmically, the method of solution is reduced to the computation of a standard basis for the ideal $I=\left\langle w_{1}, w_{2}, \ldots\right\rangle$ with respect to pure lexicographic ordering $\wp^{\prime}(\alpha) \succ \wp(\alpha) \succ k \succ \ldots$. (For example, by Buchberger's syzygy polynomials algorithm [6]);

2) Let $G=\left\{f_{1}, f_{2}, \ldots, f_{N}\right\}$ be this basis. The algebraic curve (14) and its projection on the torus (11) in algebraic form (13) are contained in $G$ if the univariate polynomial in $k$ and polynomials (13) exist;

3) If $G$ contains a polynomial free from variables $k, \wp^{\prime}(\alpha), \wp(\alpha)$, then the spectral problem (1) is not integrable in the framework of Hermite's ansatz.

Proof.

1) The standard basis always exists and Buchberger's algorithm terminates [10].

2) Taking the resultants of $w_{1}, w_{2}, \ldots$ we eliminate variables $\wp, \wp^{\prime}$ and get polynomial(s) $R(k)$. It is obvious that $R(k) \in I$. Using the divisibility

$$
\left\langle\operatorname{LT}\left(f_{1}\right), \operatorname{LT}\left(f_{2}\right), \ldots, \operatorname{LT}\left(f_{N}\right)\right\rangle=\langle\operatorname{LT}(I)\rangle
$$

and lexicographic ordering, the equality $R(k)=h(k) \widehat{f}$ has to occur for some $\widehat{f} \in G$ and $h(k) \in \mathbb{Q}(\lambda)[k]$ (possibly equal to 1 ). Therefore there exists a polynomial $\widehat{f}$ depending only on $k$. Designating $\widehat{f}(k) \equiv F(k ; \lambda)$, we obtain the curve (14). If $F(k ; \lambda)$ has a factorized form, then the algebraic curve is one of its factors. Analogously, if the polynomials (13) exist, then they necessarily belong to $G$. In the same way, an important formula - the curve as a cover of the torus (11) in a transcendental form (an equation in $\alpha$ )

$$
R\left(\wp^{\prime}(\alpha), \wp(\alpha) ; \lambda\right)=0
$$

necessarily must be contained in $G$ computed with the ordering $k \succ\left(\wp^{\prime}(\alpha) \succ\right.$ $\wp(\alpha) \succ \ldots)$, where permutations inside the brackets is allowed. Note, the order of elliptic function (15) in $\alpha$ is equal to the order $n$ of the equation (1).

3) An existence of such a polynomial implies a restriction on the spectral parameter $\lambda$ (see a demonstrative Example 5).

Note a direct link of the point 3) to a treatment of finite-gap potentials as Picard's potentials 20.

There are numerous algorithmic methods to solve this problem. Among them: the Gröbner basis method [6, 9], the method of characteristic sets, and an effective method of elimination based on the Seidenberg theory [42. We do not discuss all the modern achievements in this area. See [31] and references therein for details. Note that the reduction of the holomorphic differential $d \alpha$ to the elliptic one is derived from (13) by the formula

$$
d \alpha=\frac{d \wp(\alpha)}{\wp^{\prime}(\alpha)}=\frac{F_{k} R_{1 \lambda}-F_{\lambda} R_{1 k}}{R_{2} F_{k}} d \lambda
$$

where subscripts $k, \lambda$ denote the derivatives with respect to $k$ and $\lambda$.

\section{Canonical Form of CURVES AND holomorphic Differential}

Formulas (14 16) give a noncanonical form of the curve and holomorphic differential, i.e. expressions in the variables $(k, \lambda)$. The canonical variables $(\mu, \lambda)$ in 
the algebraic curve $\widetilde{F}(\mu, \lambda)=0$ we call variables $\lambda$ in (1) and eigenvalue $\mu$ of a commuting operator pencil

$$
\widehat{P}(\lambda) \Psi=\mu \Psi
$$

Supplementing the polar expansion of the equation (1) by the polar expansion of (17), we get the algebraic equations in variables $\left(\wp, \wp^{\prime}, k, \mu, \ldots\right)$. Again, based on the above properties of the Gröbner base, a canonical representation of the solution and all the spectral characteristics are extracted by computing the base with the ordering $\left(\wp \succ \wp^{\prime} \succ \ldots\right) \succ k \succ \mu$. Such a base contains a birational transformation between the $(k, \mu)$-variables in one direction:

$$
\mu \rightarrow k: \quad k=R_{3}(\mu ; \lambda)
$$

An inverse transformation

$$
k \rightarrow \mu: \quad \mu=R_{4}(k ; \lambda),
$$

where $R_{3,4}$ are rational functions of its arguments, is computed by the ordering $\left(\wp \succ \wp^{\prime} \succ \ldots\right) \succ \mu \succ k$.

We add a few words about the efficiency of computations. The solution of a spectral problem itself does not require the inclusion of a commuting operator (17). So, among of its polar expansions one may take (and supplement) those, containing only the $\mu$-variable. Evidently, it will enter into the polar expansion with first degree:

$$
\mu=w\left(k, \wp, \wp^{\prime} ; \lambda\right) .
$$

After the computation of the base (not including (17)), we will have the curve (14) and cover in algebraic form (13). Substituting it into the equation (20), the pair of equations for the determination of the above transformation (18, 19) is

$$
\mu=w\left(k, R_{1}(k ; \lambda), R_{2}(k ; \lambda) ; \lambda\right), \quad F(k ; \lambda)=0 .
$$

Formulae (18, 19) are obtained by computation of the bases for (21) with ordering $(k \succ \mu)$ and $(\mu \succ k)$ respectively. See Example 6 for details.

\section{Examples AND APPLications}

In this section we demonstrate the ideology of Sects. 3-4 on examples. The generality of the technique allows us to make further proofs. Let us prove that the well known potential of Treibich and Verdier (7) [40] for the equation (2) is the only possible 2-pole potential in the class

$$
u(x)=6 \wp(x)+2 \wp(x-\Omega), \quad \Omega \neq 0 .
$$

Example 1. The Treibich-Verdier potential. Parameters $\lambda, g_{2}, g_{3}$ are fixed and $\Omega$ is an unknown constant. The ansatz for the $\Psi$-function must be the following:

$$
\Psi=a_{0} \Phi(x ; \alpha, k)+a_{1} \Phi^{\prime}(x ; \alpha, k)+a_{2} \Phi(x-\Omega ; \alpha, k) .
$$

Substituting (22, 23) into (2) and equating the poles to zero, we obtain $\Psi$-function

$$
\Psi=6 \Phi(-\Omega ; \alpha, k) \Phi^{\prime}(x ; \alpha, k)-\left(3 k^{2}-3 \wp_{\alpha}-2 \wp_{\Omega}-\lambda\right) \Phi(x-\Omega ; \alpha, k)
$$


and a system of five polynomials:

$$
\begin{aligned}
w_{1}= & 2\left(\wp_{\alpha}-\wp_{\Omega}\right) k^{3}+3\left(\wp_{\Omega}^{\prime}-\wp_{\alpha}^{\prime}\right) k^{2}+2\left(\wp_{\alpha}-\wp_{\Omega}\right)\left(3 \wp_{\alpha}-\lambda-2 \wp_{\Omega}\right) k+ \\
& +\left(6 \wp_{\Omega}-\wp_{\alpha}\right) \wp_{\alpha}^{\prime}-\left(\wp_{\Omega}^{\prime}-\wp_{\alpha}^{\prime}\right) \lambda-\left(7 \wp_{\alpha}-2 \wp_{\Omega}\right) \wp_{\Omega}^{\prime} \\
w_{2}= & 3 k^{3}-\left(9 \wp_{\alpha}-4 \wp_{\Omega}+\lambda\right) k+3 \wp_{\alpha}^{\prime}+3 \wp_{\Omega}^{\prime} \\
w_{3}= & 3 k^{4}-2\left(9 \wp_{\alpha}-14 \wp_{\Omega}-\lambda\right) k^{2}+12\left(\wp_{\Omega}^{\prime}+\wp_{\alpha}^{\prime}\right) k-9 \wp_{\alpha}^{2}- \\
& -2\left(14 \wp_{\Omega}+\lambda\right) \wp_{\alpha}+12 \wp_{\Omega}^{2}-8 \lambda \wp_{\Omega}-\lambda^{2} \\
w_{4}= & \wp_{\alpha}^{\prime 2}-4 \wp_{\alpha}^{3}+g_{2} \wp_{\alpha}+g_{3} \\
w_{5}= & \wp_{\Omega}^{\prime 2}-4 \wp_{\Omega}^{3}+g_{2} \wp_{\Omega}+g_{3},
\end{aligned}
$$

where we used the addition theorems for elliptic functions, the important equality

$$
\Phi(\Omega ; \alpha, k) \Phi(-\Omega ; \alpha, k)=\wp_{\alpha}-\wp_{\Omega},
$$

and designated $\wp_{\alpha} \equiv \wp(\alpha), \wp_{\Omega} \equiv \wp(\Omega)$, etc. A common factor $\wp_{\alpha}-\wp_{\Omega}$ was removed in polynomial $w_{3}$ because it leads to the contradiction: $\alpha=\Omega\left(a_{2}=\infty\right)$. The system (24) generates the ideal

$$
\left\langle w_{1}, w_{2}, w_{3}, w_{4}, w_{5}\right\rangle \in \mathbb{Q}\left(\lambda, g_{2}, g_{3}\right)\left[k, \wp_{\alpha}^{\prime}, \wp_{\alpha}, \wp_{\Omega}^{\prime}, \wp_{\Omega}\right] .
$$

Note, the same ideal $\left\langle w_{1}, \ldots, w_{5}\right\rangle$ in the ring $\mathbb{Q}\left(\lambda, g_{2}, g_{3}, \wp_{\Omega}\right)\left[k, \wp_{\alpha}^{\prime}, \wp_{\alpha}, \wp_{\Omega}^{\prime}\right]$ leads to the just mentioned condition $\wp_{\alpha}-\wp_{\Omega}=0$. Therefore $\Omega$ is not arbitrary. Computing the minimal reduced Gröbner basis for (25) with pure lexicographic ordering $\wp_{\alpha}^{\prime} \succ$ $\wp_{\alpha} \succ k \succ \wp_{\Omega}^{\prime} \succ \wp_{\Omega}$, we obtain 8 polynomials, some of them having a factorized form. If some of the factors do not depend on $\lambda$, we obtain restrictions on $\wp_{\Omega}^{\prime}$ and $\wp_{\Omega}$ equating these factors to zero. There are four such polynomials:

$$
\begin{aligned}
& G_{1}=\wp_{\Omega}^{\prime}\left(\left(\lambda^{3}-4 g_{2} \lambda-16 g_{3}\right) k+\left(3 \lambda^{2}-4 g_{2}\right) \wp_{\Omega}^{\prime}\right) M, \\
& G_{2}=\wp_{\Omega}^{\prime}\left(16 \wp_{\Omega}^{\prime} k+3 \lambda^{2}-4 g_{2}\right) M, \\
& G_{3}=\left(4 \wp_{\Omega}^{3}-g_{2} \wp_{\Omega}-g_{3}\right)\left(4 \wp_{\Omega}-\lambda\right) M, \\
& G_{4}=\wp_{\Omega}^{\prime}\left(4 \wp_{\Omega}-\lambda\right) M,
\end{aligned}
$$

where the multiplier $M$ denotes $3 k^{2}-\lambda-5 \wp_{\Omega}$. The equation $M=0$ yields the trivial result $\wp(\alpha)=\wp(\Omega)$. It is checked by recomputing the base (24) with an additional polynomial $M$. Further, $\Omega$ must not depend on $\lambda(!)$. Therefore, the only solution for $\Omega$ is defined by the equation

$$
\wp^{\prime}(\Omega)=0 \Longrightarrow \Omega=\omega_{1}, \omega_{2}, \omega_{3},
$$

where $\omega_{j}$ are the half-periods of elliptic functions. Substituting $\wp_{\Omega}^{\prime}=0, \wp_{\Omega}=e_{1}$, $g_{2}=4\left(e_{1}^{2}+e_{1} e_{2}+e_{2}^{2}\right), g_{3}=-4 e_{1} e_{2}\left(e_{1}+e_{2}\right)$ into (24) and recomputing the basis with respect to the ordering $\wp_{\alpha}^{\prime} \succ \wp_{\alpha} \succ k$, we obtain the well known algebraic curve of genus 2 and all algebraic-geometric objects [2, 33]. For classification results of the Treibich-Verdier potentials and other elliptic ones, see [33], the appendix in [15] and the most recent results in the review [36].

Example 2. As a preliminary, we shall consider equation (3) with potential

$$
u(x)=6 \wp(x)+6 \wp(x-\Omega)
$$

and the restriction $g_{2}=0$. As before, we have the ansatz for the $\Psi$-function

$$
\Psi=\Phi(x ; \alpha, k)+a_{1} \Phi(x-\Omega ; \alpha, k)
$$


and the original basis of the ideal is generated by 5 polynomials. Computing the Gröbner basis $G$, we obtain a system of 8 polynomials. Only two of them have a factorized form.

$$
\begin{aligned}
& G_{1}=\left(64 \lambda^{3} k^{3}-27\left(\lambda^{2}+16 g_{3}\right)^{2}\right)\left(\wp_{\Omega}^{\prime} k-3 \wp_{\Omega}^{2}\right), \\
& G_{2}=\left(64 \lambda^{3} k^{3}-27\left(\lambda^{2}+16 g_{3}\right)^{2}\right)\left(\left(4 \wp_{\Omega}^{3}-g_{3}\right) k-3 \wp_{\Omega}^{\prime} \wp_{\Omega}^{2}\right) .
\end{aligned}
$$

The nontrivial solution will take place if and only if $(k, \lambda)$ be coordinates of the algebraic curve which is the first factor in $G_{1}, G_{2}$. In the next example we rule out the condition $g_{2}=0$.

Example 3. If $g_{2}$ is free, the straightforward computing of the basis is unsuccessful. Indeed, the Gröbner base method is universal and therefore it can be ineffective in some special cases. But our interest is only with the zero structure of the polynomial system. Thus, the characteristic sets method 41] is the best approach in this case. Under the ordering $\wp_{\Omega} \prec \wp_{\Omega}^{\prime} \prec k \prec \wp_{\alpha} \prec \wp_{\alpha}^{\prime}$, the characteristic set has the form

$$
\begin{aligned}
f_{1}= & \left(64\left(k \lambda+g_{2}\right)\left(k \lambda-2 g_{2}\right)^{2}-27\left(\lambda^{2}+16 g_{3}\right)^{2}\right) k M, \\
f_{2}= & \left(8\left(k \lambda-2 g_{2}\right) \wp_{\alpha}-8 k^{3} \lambda+16 g_{2} k^{2}+3 \lambda^{2}+48 g_{3}\right) . \\
& \left(4 \wp_{\Omega}^{\prime} k+g_{2}-12 \wp_{\Omega}^{2}\right) k, \\
f_{3}= & \left(32\left(k \lambda-2 g_{2}\right) \lambda^{3} \wp_{\alpha}^{\prime}-192 g_{2}^{2} k^{2} \lambda^{2}+\right. \\
& +\left(\lambda^{4}-288 g_{3} \lambda^{2}-6912 g_{3}^{2}+2^{8} g_{2}^{3}\right) k \lambda- \\
& \left.-g_{2}\left(11 \lambda^{4}+864 g_{3} \lambda^{2}+6912 g_{3}^{2}-2^{8} g_{2}^{3}\right)\right) k M, \\
f_{4}= & \wp_{\Omega}^{\prime 2}-4 \wp_{\Omega}^{3}+g_{2} \wp_{\Omega}+g_{3},
\end{aligned}
$$

where

$$
\begin{aligned}
M \equiv & 64 \wp_{\Omega}^{\prime} \wp_{\Omega} k^{2}-4\left(3 \wp_{\Omega}^{\prime} \lambda-16 g_{2} \wp_{\Omega}-12 g_{3}+96 \wp_{\Omega}^{3}\right) k+ \\
& +3\left(12 \wp_{\Omega}^{2}-g_{2}\right)\left(\lambda+4 \wp_{\Omega}^{\prime}\right) .
\end{aligned}
$$

Factorisation shows that the variable $\Omega$ is separated in polynomials $f_{1,2,3}$. Therefore $f_{1}$ gives an algebraic curve independent of $\Omega$ :

$$
64\left(k \lambda+g_{2}\right)\left(k \lambda-2 g_{2}\right)^{2}=27\left(\lambda^{2}+16 g_{3}\right)^{2} .
$$

The polynomials $\left\{f_{2}, f_{3}\right\}$ are an algebraic form of the cover (13). However, the genus of the curve (28) is unity and we have a cover of a torus by a torus. Hence, if moduli of both tori are equal, then there is a one-to-one correspondence between the global parameter $\alpha$ of the torus (11) and the global parameter $\tau$ of the torus (28). The next step is to find it. After the birational change of variables $(k, \lambda) \leftrightarrow(y, x)$ :

$$
k=\frac{3 y^{2}+2 g_{2} x+3 g_{3}}{4 y x}, \quad \lambda=4 y
$$

we obtain the canonical form of the curve (28) as $y^{2}=4 x^{3}-g_{2} x-g_{3}$ with an obvious uniformisation and the equality $\alpha=2 \tau$. The final solution of the problem 
$(3,26)$ is as follows:

$$
\begin{gathered}
\Psi(x ; \lambda)=a \Phi(x ; 2 \tau, k)+\Phi(\Omega ; 2 \tau, k) \Phi(x-\Omega ; 2 \tau, k), \quad \lambda=-4 \wp^{\prime}(\tau), \\
a=\zeta(2 \tau+\Omega)-2 \zeta(\tau)-\zeta(\Omega), \quad k=2 \zeta(\tau)-\zeta(2 \tau) .
\end{gathered}
$$

The passage to the limit $\tau \rightarrow \omega_{j}$ in (29) leads to the solution under the condition $\lambda=0$ :

$$
\Psi(x ; \lambda=0)=C_{1}(\zeta(x)-\zeta(x-\Omega))+C_{2} .
$$

An attempt to integrate the more general potential

$$
u(x)=6 \wp\left(x-\Omega_{1}\right)+6 \wp\left(x-\Omega_{2}\right)+A
$$

with a nonzero constant $A$ failed. However, this point has an explanation in the theory of nonlinear partial differential equations. Indeed, the spectral problem (3) is associated with the Sawada-Kotera (SK) equation [32]

$$
u_{t}=u_{x x x x x}-5 u u_{x x x}-5 u_{x} u_{x x}+5 u^{2} u_{x}
$$

By assuming that the poles $\Omega_{1,2}$ depend on time $t$, one obtains an isospectral deformation of this potential. This simple calculation yields the stationary solution of (30)

$$
u(x, t)=6 \wp(x-c t)+6 \wp(x-c t-\Omega)+A
$$

with the conditions

$$
c+12 g_{2}+5 A^{2}+60 A \wp(\Omega)=0, \quad A \wp^{\prime}(\Omega)=0 .
$$

Therefore $\left(\Omega=\omega_{j}\right.$ and $A$ is free) or $(A=0$ and $\Omega$ is free). In the both cases we obtain a restriction on a velocity $c$ of two cnoidal waves. See an example in [39] for the case $A=0$. Recently, Conte and Musette obtained a similar result [30, formula (84)] and revealed a remarkable more general solution in an old paper of Chazy [7] in the context of the Painlevé analysis:

$$
\begin{gathered}
u(x, t)=6 \wp\left(x-c t-\Omega ; g_{2}, g_{3}\right)+6 \wp\left(x-\tilde{c} t-\widetilde{\Omega} ; \widetilde{g}_{2}, \widetilde{g}_{3}\right), \\
c=3 g_{2}-15 \widetilde{g}_{2}, \quad \widetilde{c}=3 \widetilde{g}_{2}-15 g_{2} .
\end{gathered}
$$

Strictly speaking, Chazy's solution [7, p. 380] corresponds to the stationary equation (30) and therefore to the case $\widetilde{g}_{2}=g_{2}(\widetilde{c}=c)$ in (31). One can show that the potential (31) is the stationary solution of a linear combination of the equation (30) and higher SK-equation of the 7-th order

$$
\begin{aligned}
u_{t} & =u_{7 x}- \\
& -7\left(u u_{5 x}+2 u_{x} u_{4 x}+3 u_{x x} u_{x x x}-2 u^{2} u_{x x x}-6 u u_{x} u_{x x}-u_{x}^{3}+\frac{4}{3} u^{3} u_{x}\right) .
\end{aligned}
$$

Sect. 7 contains additional information for this potential. We do not enumerate other 1-pole elliptic potentials $u(x)=A \wp(x)+B$ for the equation (3). For example, one of them is $u=30 \wp(x) \pm 3 \sqrt{3 g_{2}}$ (see also [5]).

Example 4. Let us consider a general 1-pole elliptic potential for the equation (位)

$$
\Psi^{\prime \prime \prime}-(a \wp(x)+d) \Psi^{\prime}-\left(b \wp^{\prime}(x)+c \wp(x)\right) \Psi=\lambda \Psi
$$

in the framework of the ansatz

$$
\Psi=\Phi(x ; \alpha, k) .
$$

Using the above techniques in the ring $\mathbb{Q}(a, b, c, d)\left[\wp_{\alpha}, \wp_{\alpha}^{\prime}, k\right]$ we do not get the solution: $I=\langle 1\rangle$. Therefore $(a, b, c, d)$ have to depend on each other. After calculations 
in the ring $\mathbb{Q}(a, b, c)\left[\wp_{\alpha}, \wp_{\alpha}^{\prime}, k, d\right]$ we determine step by step the constants $(a, b, c, d)$ and get the following. The first polynomial in a base is

$$
-8 b^{3}(2 b-3)^{3} \lambda^{2}-4 c(2 b-3)^{2}\left(4 d b^{3}-12 b^{2} d-b c^{2}+6 c^{2}\right) \lambda+\cdots=0 .
$$

Equating to zero the coefficients in front of $\lambda^{2}, \lambda$ we obtain

$$
b=\frac{3}{2}, \quad \text { or } \quad b=0 .
$$

In these cases we will have respectively

$$
3 d-c^{2}=0, \quad\left(216 \lambda+c^{3}-36 c d\right) c^{3}=0 .
$$

Therefore $\left(b=3 / 2, d=c^{2} / 3\right)$ or $b=c=0$. In the first case we have

$$
\Psi^{\prime \prime \prime}-3\left(\wp(x)+c^{2}\right) \Psi^{\prime}-\left(\frac{3}{2} \wp^{\prime}(x)+3 c \wp(x)\right) \Psi=\lambda \Psi, \quad \Psi(x ; \lambda)=\Phi(x ; \alpha, c) .
$$

The nonramified cover of the torus (15) of genus $g=1$ is

$$
\wp^{\prime}(\alpha)-6 c \wp(\alpha)+2 \lambda+4 c^{3}=0 .
$$

$c$ is an arbitrary constant and the condition $g_{2}=0$ [28, example 3.10] does not appear. Note, there is no such restriction in the Halphen equation (6) with $n=5$ as in [14]. It appears only for $n=4$ [16]. The second case is known [28]:

$$
\Psi^{\prime \prime \prime}-(6 \wp(x)+d) \Psi^{\prime}=\lambda \Psi
$$

$$
\left.108 \lambda \wp^{\prime}(\alpha)+36\left(d^{2}-3 g_{2}\right) \wp(\alpha)+27 \lambda^{2}-108 g_{3}-4 d^{3}=0 \quad \text { (genus } g=2\right) \text {. }
$$

See [3] for an application of this potential.

Note that both cases can be found in [27, t. III: pp. 372, 522] in Jacobian functions and [18, III/IV: pp. 460, 462] in Weierstrassian functions. No other possibilities exist. The same technique is applicable to other ansatzs. The next one is a nontrivial example along these lines.

Example 5. The equation (33) in the framework of the ansatz

$$
\Psi=a_{0} \Phi(x ; \alpha, k)+\Phi^{\prime}(x ; \alpha, k) .
$$

As a consequence of corresponding indicial equation (37) with $\nu=2$, without loss of generality we get $b=12-a$ in (33). Solutions for $a, c, d$ must not depend on $\lambda$ and $k$. One solution suggests itself. Indeed, the first polynomial in the original base has the form

$$
(a-12)\left((a-18)\left(k^{2}-\wp_{\alpha}\right)+2 c k\right)+2(a-18) d+c^{2}=0 .
$$

With $a=12$, this polynomial does not depend on $k, \alpha$ and we get (after the replacement $c \rightarrow 12 c$ )

$$
a=12, \quad b=0, \quad d=12 c^{2}, \quad a_{0}=k-2 c .
$$

Moreover, the ideal in the ring $\mathbb{Q}\left(\lambda, c, g_{2}, g_{3}\right)\left[\wp_{\alpha}, \wp_{\alpha}^{\prime}, k\right]$ is not equal to $\langle 1\rangle$ and therefore, $c$ is an arbitrary constant. Thus, the equation (33) and its solution take the form

$$
\begin{gathered}
\Psi^{\prime \prime \prime}-12\left(\wp(x)+c^{2}\right) \Psi^{\prime}-12 c \wp(x) \Psi=\lambda \Psi \\
\Psi(x ; \lambda)=\Phi^{\prime}(x ; \alpha, k)-2 c \Phi(x ; \alpha, k) .
\end{gathered}
$$


We do not give here the large formulae for the cover (13), or the 4-sheet cover in the form (15) and write only a skeleton of the non-hyperelliptic trigonal algebraic curve (14) of genus $g=3$

$$
64\left(\lambda^{2}+32 c^{3} \lambda+2^{8} c^{6}-108 g_{2} c^{2}\right)\left(\lambda-11 c^{3}\right) k^{3}+(\cdots) k^{2}+(\cdots) k+(\cdots)=0,
$$

where $(\cdots)$ designate some polynomials in $\lambda, c, g_{2}, g_{3}$ with integer coefficients [5]. Under $c=0$ we arrive at the case (26) with $\Omega=0$.

The higher ansatzs (9) are investigated in a similar manner. Indeed, by Frobenius theory, if $\Psi$ has the expansion $\Psi=x^{-\nu}+\cdots$, then $a, b$ satisfy the determining equation

$$
-\nu(\nu+1)(\nu+2)+a \nu+2 b=0 .
$$

A natural question appears: under what parameters $(a, b)$ does the equation (37) have integral solutions for $\nu$ ? One of solutions is Halphen's equation (5). It corresponds to $2 b=a$ and (37) is reduced to

$$
a=n^{2}-1 \quad(n \equiv \nu+1) .
$$

As in the previous example we can list all the possible cases for the ansatz (35). Indeed, assuming $b=12-a$ and $(a, d, c)$ to be arbitrary, the origin base contains three polynomials [5]. The first and second of them are linear in $\wp, \wp^{\prime}$. Solving them and substituting into the base again, we obtain the remaining polynomial in $(\lambda, k)$ :

$$
-4(a-12)^{2}(a-18)^{2}((a-6)(a-18) k+c(a-9)) \lambda+(\cdots) k+(\cdots)=0,
$$

where dots denote a polynomial in $\left(a, c, d, g_{2}, g_{3}\right)$. It must be zero for all values of $\lambda$. Splitting it in $\lambda$ we get two linear polynomials in $k$. Their compatibility condition is the polynomial

$$
(a-6)(a-8)\left(108 c^{4}-72(a-18)^{2} d c^{2}+(a-18)^{4}\left(12 d^{2}-(a-12)^{2} g_{2}\right)\right)=0
$$

and solution for $k$

$$
k=-\frac{(a-9) c}{(a-6)(a-18)} .
$$

The verifying of Weierstrass's relation (11) yields a polynomial in $\lambda$

$$
(a-6)^{3}(a-12)^{3}(a-18)^{6} \lambda^{2}+(\cdots) \lambda+(\cdots)=0 .
$$

Under $a \neq 6,12,18$ we arrive at the point 3 ) of the Proposition. Therefore, only three possibilities exist: $a=6,12,18$. The corresponding final solutions for the variables $\left(\wp^{\prime}, \wp, k\right)$ are obtained separately: by recomputing the base. Thus, besides (36), we have the following integrable potentials (note a misprint $\wp(x)$ instead of $\wp^{\prime}(x)$ in one of the formulae in [5])

$\Psi^{\prime \prime \prime}-(18 \wp(x)+d) \Psi^{\prime}+6 \wp^{\prime}(x) \Psi=\lambda \Psi, \quad \Psi^{\prime \prime \prime}-(6 \wp(x)+d) \Psi^{\prime}-6 \wp^{\prime}(x) \Psi=\lambda \Psi$.

See [38] for solutions of the generalized Halphen equation (6) and [5] for details of the Example 5.

It should perhaps be noted here that the example $(36)$ is the generalization $c \neq 0$ of the first nontrivial case $n=-3$ in a series of other Halphen's equations [26. p. 554]

$$
w^{\prime \prime \prime}-\frac{4}{3} n^{2} w^{\prime} \wp(z)-\frac{2}{27} n(n+3)(4 n-3) w \wp^{\prime}(z)=0
$$


without a spectral parameter Notation as in 18, III/IV: Ex.15, p.464]. Indicial equation (37) for the example (38) becomes

$$
(3 \nu+2 n)(3 \nu+2 n+6)(3 \nu-4 n+3)=0
$$

and $(n+3)(n+6)(4 n-9)=0$ for the ansatz (35) $(\nu=2)$.

Example 6. Halphen's equation (6) with $n=5$. Here we display only the final formulae in the context of Sect. 4:

- The commuting operator pencil (17):

$$
\lambda \Psi^{\prime \prime}-14\left(4 \wp(x)^{2}-g_{2}\right) \Psi^{\prime}+16\left(7 \wp^{\prime}(x)-\lambda\right) \wp(x) \Psi=\mu \Psi ;
$$

- The polynomial (20):

$6 \mu-56 k^{5}+560 \wp_{\alpha} k^{3}-20\left(28 \wp_{\alpha}^{\prime}-\lambda\right) k^{2}+168\left(5 \wp_{\alpha}^{2}-g_{2}\right) k-4\left(28 \wp_{\alpha}^{\prime}+5 \lambda\right) \wp_{\alpha}=0 ;$

- The birational transformation (18, 19), which is quadratic in $(k, \mu)$ :

$$
\begin{gathered}
\mu=\frac{32}{49} \frac{\left(2\left(\lambda^{2}-392 g_{3}\right) k \lambda+7\left(5 \lambda^{2}-784 g_{3}\right) g_{2}\right)\left(\left(\lambda^{2}-392 g_{3}\right) k-21 g_{2} \lambda\right)}{\lambda^{4}-208 g_{3} \lambda^{2}+3136\left(g_{2}^{3}+4 g_{3}^{2}\right)}, \\
k=\frac{7}{8} \frac{\mu^{2}-4 g_{2}\left(5 \lambda^{2}-784 g_{3}\right)}{\left(\lambda^{2}-392 g_{3}\right) \lambda}
\end{gathered}
$$

- The canonical form of the algebraic curve of genus 4 (see also [38]):

$\widetilde{F}(\mu, \lambda): \quad \mu^{3}-4 g_{2}\left(11 \lambda^{2}-784 g_{3}\right) \mu-\lambda^{5}+208 g_{3} \lambda^{3}-3136\left(g_{2}^{3}+4 g_{3}^{2}\right) \lambda=0 ;$

- The 8-sheet cover in the form (15):

$2^{8}\left(\lambda^{2}-392 g_{3}\right)^{3} \lambda \wp^{\prime}(\alpha)-2^{8} 49 g_{2}\left(\lambda^{2}+112 g_{3}\right)\left(\lambda^{2}-392 g_{3}\right)^{2} \wp(\alpha)+\lambda^{8}-\cdots=0$.

Note that both this cover and its algebraic form (13) are the expansive expressions, whereas the reduced holomorphic differential (16) in the variables $(k, \lambda)$ and $(\mu, \lambda)$ is given by the simple formulae:

$$
\frac{d \wp(\alpha)}{\wp^{\prime}(\alpha)}=\frac{-8\left(\lambda^{2}-56 g_{3}\right)}{3 \mu^{2}-4 g_{2}\left(11 \lambda^{2}-784 g_{3}\right)} d \lambda=\frac{-7\left(\lambda^{2}-56 g_{3}\right)}{\left(\lambda^{2}-392 g_{3}\right)\left(3 \lambda k+14 g_{2}\right)} d \lambda .
$$

Analogs of the above formulae are derived for all other examples in the paper.

Example 7. The 2-pole potential for the equation (4) with ansatz

$$
\Psi=\Phi(x ; \alpha, k)+a_{1} \Phi(x-\Omega ; \alpha, k) .
$$

The general 2-pole elliptic potentials contain many parameters - multipliers before the $\wp^{\prime}, \wp, \zeta$-functions. We do not give their exhaustive classification and consider only the most interesting case

$$
\begin{aligned}
\Psi^{\prime \prime \prime} & -3(\wp(x)+\wp(x-\Omega)-\wp(A)) \Psi^{\prime}- \\
& -\frac{3}{2}\left(\wp^{\prime}(x)+\wp^{\prime}(x-\Omega)+B \wp(x)-B \wp(x-\Omega)\right) \Psi=\lambda \Psi .
\end{aligned}
$$

By virtue of the Proposition, the Gröbner base contains all the information about the solution, i.e. all the following formulae. As before we obtain that $\Omega, A$ are arbitrary constants and

$$
B=2 \sqrt{\wp(\Omega)-\wp(A)} .
$$

\footnotetext{
${ }^{1}$ The example (38) was revealed by E. Previato
} 
The parameters $k$ and $\lambda$ as meromorphic functions are related by a algebraic equation of the genus 2 independent of $\Omega$ (compare with [34]):

$$
2 \lambda k^{3}+\left(3 \wp_{A}^{2}-g_{2}\right) k^{2}-3 \wp_{A} \lambda k-\frac{1}{4} \lambda^{2}+\wp_{A}^{\prime 2}=0 .
$$

The equation (40) can be realized as a 2 -sheet cover of a torus in the form (15)

$$
\lambda \wp^{\prime}(\alpha)+\left(3 \wp_{A}^{2}-g_{2}\right) \wp(\alpha)+\frac{1}{4} \lambda^{2}+\wp_{A}^{3}-g_{3}=0 .
$$

The algebraic form of cover (13) has the form

$$
\wp(\alpha)=k^{2}+\wp_{A}, \quad \wp^{\prime}(\alpha)=\left(g_{2}-3 \wp_{A}^{2}\right) \frac{k^{2}}{\lambda}-\frac{\wp_{A}^{\prime 2}}{\lambda}-\frac{\lambda}{4} .
$$

\section{Solutions of integrable PDE's}

The spectral problem (1, 39) corresponds to the Boussinesq equation

$$
3 u_{t t}=\left(2 u^{2}-u_{x x}\right)_{x x},
$$

and the arbitrariness of $\Omega$ means the existence of an isospectral deformation of the potential

$$
u(x, t)=3 \wp\left(x-\Omega_{1}(t)\right)+3 \wp\left(x-\Omega_{2}(t)\right)-3 \wp_{A} .
$$

Substituting the 2-gap ansatz (42) in (41), we get the well known system of pairwiseinteracting particles $\Omega_{1,2}(t)$ of the Calogero-Moser system type [29] with a repulsion potential and immovable center of mass. Integration leads to an equation for $\Omega_{1}(t)$ :

$$
\dot{\Omega}_{1}^{2}=4 \wp\left(2 \Omega_{1}-c\right)-4 \wp_{A}, \quad \Omega_{2}=c-\Omega_{1} .
$$

Using the uniformisation of the corresponding elliptic curve, we obtain the explicit solution

$$
\begin{gathered}
\Omega_{1}(t)=\frac{1}{2} \wp^{-1}\left(\widetilde{\zeta}(\tau+\nu)-\widetilde{\zeta}(\tau-\nu)-\widetilde{\zeta}(2 \nu)+\frac{1}{4} \wp_{A}\right)+\frac{c}{2}, \\
\nu= \pm \frac{1}{2} \widetilde{\wp}^{-1}\left(\frac{\wp_{A}^{2}}{16}+\frac{g_{2}}{24}\right), \quad \widetilde{\wp}^{\prime}(2 \nu)=-\frac{1}{32}\left(\wp_{A}^{3}-g_{2} \wp_{A}+2 g_{3}\right),
\end{gathered}
$$

where $\tau=8\left(t-t_{0}\right)$ and $c, t_{0}$ are arbitrary constants. An implicit form of this solution with $c=0$ in terms of Jacobi's sn, cn, dn-functions is given in [19] and in the earlier citation [8] in the context of solutions of the Kadomtsev-Petviashvili equation. In (43), the elliptic integral $\wp^{-1}$ is calculated with invariants $g_{2}, g_{3}$, and the $\widetilde{\zeta}, \widetilde{\wp}^{-1}$-functions with invariants

$$
\widetilde{g}_{2}=\frac{1}{16}\left(g_{2} \wp_{A}^{2}+3 g_{3} \wp_{A}+\frac{g_{2}^{2}}{12}\right), \quad \widetilde{g}_{3}=-\frac{g_{3}}{2^{8}}\left(\wp_{A}^{\prime 2}+2 g_{3}\right)+\frac{g_{2}^{3}}{2^{7} 3^{3}}-\frac{g_{2} \widetilde{g}_{2}}{24} .
$$

The reduction case $B=0$ in (39) corresponds to the Kaup-Kupershmidt (KK) equation

$$
u_{t}=u_{x x x x x}-5 u u_{x x x}-\frac{25}{2} u_{x} u_{x x}+5 u^{2} u_{x}
$$

and its stationary solution

$$
u(x, t)=3 \wp(x-c t)+3 \wp(x-c t-\Omega)-3 \wp(\Omega),
$$

but the velocity $c$ depends on the distance between poles:

$$
c=3 g_{2}-45 \wp^{2}(\Omega) \text {. }
$$


The generalization of (45) in a similar way as the solution (31) is

$$
u(x, t)=-12\left(\wp_{1}+\wp_{2}\right)+3\left(\frac{\wp_{1}^{\prime}-\wp_{2}^{\prime}}{\wp_{1}-\wp_{2}}\right)^{2},
$$

where

$$
\wp_{1} \equiv \wp\left(x-c t-\Omega ; g_{2}, g_{3}\right), \quad \wp_{2} \equiv \wp\left(x-c t-\widehat{\Omega} ; g_{2}, \widehat{g}_{3}\right), \quad c=-12 g_{2} .
$$

Here $\Omega, \widehat{\Omega}, g_{2}, g_{3}, \widehat{g}_{3}$ are five arbitrary constants. Using a connection between the $\operatorname{SK}(u)$ - and $\operatorname{KK}(w)$-equations and the Tzitzeica equation

$$
\phi_{x t}=e^{\phi}-e^{-2 \phi}
$$

with the Fordy-Gibbons equation 17

$$
v_{t}=v_{x x x x x}-5\left(v_{x} v_{x x x}+v^{2} v_{x x x}+v_{x x}^{2}+v_{x}^{3}+4 v v_{x} v_{x x}-v^{4} v_{x}\right)
$$

via the Miura transformations

$$
u=v^{2}-v_{x}, \quad w=v^{2}+2 v_{x}, \quad u=\phi_{x x}+\phi_{x}^{2}
$$

we obtain stationary solutions for the $v$-function

$$
v(x, t)=\frac{\wp_{1}^{\prime}-\wp_{2}^{\prime}}{\wp_{1}-\wp_{2}}
$$

Non-stationary solution of the equation (47) has the form

$$
\phi(x, t)=\ln 2 c+\ln \left(\wp\left(x+c t-\Omega ; g_{2}, g_{3}\right)-\wp\left(x-c t-\widehat{\Omega} ; g_{2}, \widehat{g}_{3}\right)\right)
$$

with the restriction: $4\left(\widehat{g}_{3}-g_{3}\right) c^{3}=1$. The details of calculations 43 are expounded in [5] and the formula (46), $\Psi$-function for the potentials (31, 46) in 5. 4 . 39 .

\section{Concluding Remarks AND Discussion}

The investigation of elliptic solitons can be automated by a polynomial techniques. The Gröbner base method provides an unified approach to the solution of related problems. The technique suggested with minor modifications is extended to matrix spectral problems.

As the Examples 2-3 and $\mathbf{5}$ show, the algebraic curves can be degenerate (multiply roots of discriminant).

The general case in Example $\mathbf{7}$ for the equation (4)

$$
\begin{aligned}
& u(x)=a \wp(x)+b \wp(x-\Omega)+c \zeta(x)-c \zeta(x-\Omega), \\
& v(x)=d \wp^{\prime}(x)+e \wp^{\prime}(x-\Omega)+f \wp(x)+g \wp(x-\Omega)+h \zeta(x)-h \zeta(x-\Omega)
\end{aligned}
$$

requires additional research.

To all appearances, the example (36) has to fit into the hierarchy of higher Boussinesq equations, studied in full in [12, 13. Multi-pole potentials are investigated by involving the addition theorem for the $\Phi$-function:

$$
\Phi(x+z ; \alpha, k)=\frac{1}{2} \frac{\Phi(x ; \alpha, k) \Phi(z ; \alpha, k)}{\wp_{x}-\wp_{z}}\left(\frac{\wp_{\alpha}^{\prime}+\wp_{x}^{\prime}}{\wp_{\alpha}-\wp_{x}}-\frac{\wp_{\alpha}^{\prime}+\wp_{z}^{\prime}}{\wp_{\alpha}-\wp_{z}}\right) .
$$

A natural assumption suggests itself: all the potentials, obtained by the above method, are finite-gap ones. At least, by construction, all such potentials belong to the set of exact integrable (explicit $\Psi$ [39]) and the $\Psi$-function is a single-value function on a Riemann surface of the algebraic curve $F(k, \lambda)=0$ and meromorphic 
function (in $x$ ) for all values of $\lambda$ (Picard's theorem [20, 22]). Note that remaining linear independent solutions for the $\Psi$-function are got by choosing of $k$-branch of algebraic equation (14).

If the assumption is valid, then the potentials for the spectral problems (2-11) are free of residues (a consequence of $\Theta$-formulas), and therefore ansatzs for the multipole potentials (say (48)) do not have to involve the $\zeta$-functions. This strongly decreases the number of parameters and the computational task.

The potential (31)

$$
u=6 \wp\left(x-\Omega ; g_{2}, g_{3}\right)+6 \wp\left(x-\widetilde{\Omega} ; \widetilde{g}_{2}, \widetilde{g}_{3}\right)
$$

with arbitrary invariants $g_{2}, g_{3}, \widetilde{g}_{2}, \widetilde{g}_{3}$ is a finite-gap one for the equation (3), but its spectral characteristics can not be obtained in the framework of elliptic soliton theory. The corresponding commuting operator pencil is derived with the help of the equation (32) and takes the form

$$
\begin{aligned}
& \left(9\left(u-c_{1}\right) \lambda-3 u^{\prime \prime \prime}+6 u u^{\prime}+c_{1} u^{\prime}\right) \Psi^{\prime \prime}- \\
& -\left(27 \lambda^{2}+9 u^{\prime} \lambda-u^{(\mathrm{IV})}-3 u^{\prime 2}+\frac{4}{3} u^{3}-c_{1} u^{\prime \prime}-c_{1} u^{2}+27 c_{2}\right) \Psi^{\prime}+ \\
& +6 \lambda\left(u^{\prime \prime}-u^{2}+c_{1} u\right) \Psi=\mu \Psi .
\end{aligned}
$$

Hence, the canonical form $\widetilde{F}(\mu, \lambda)=0$ of the associated trigonal curve of genus $g=4$ is obtained by elimination of $\Psi$ :

$$
\begin{aligned}
& a^{3} \mu^{3}+3^{5}\left(36 a^{2} b \lambda^{4}-\left(a^{5}-16 g_{2} a^{4}+16 g_{2}^{2} a^{3}+192\left(b-3 g_{3}\right) a^{2} b+192 g_{2} a b^{2}\right) \lambda^{2}+\right. \\
& \left.+48\left(g_{3} a^{5}-g_{2} a^{4} b+4 a^{2} b^{3}\right)\right) \mu+3^{6}\left(27 a^{3} \lambda^{7}-216\left(a^{3} b-4 g_{3} a^{3}+2 g_{2} a^{2} b+8 b^{3}\right) \lambda^{5}-\right. \\
& -2\left(a^{6}+30 g_{2} a^{5}-96 g_{2}^{2} a^{4}-8\left(45 b^{2}-216 g_{3} b-8 g_{2}^{3}+432 g_{3}^{2}\right) a^{3}+\right. \\
& \left.+576\left(b+6 g_{3}\right) g_{2} a^{2} b-2^{8} 9 g_{2}^{2} a b^{2}-2^{8} 3^{3}\left(b^{4}-2 g_{3} b^{3}\right)\right) \lambda^{3}- \\
& \left.-288\left(a^{3}-2 g_{2} a^{2}+24 b^{2}\right)\left(g_{3} a^{3}-g_{2} a^{2} b+4 b^{3}\right) \lambda\right)=0, \quad a \equiv g_{2}-\widetilde{g}_{2}, \quad b \equiv g_{3}-\widetilde{g}_{3}
\end{aligned}
$$

and the corresponding $\Psi$-function is given by the expression

$$
\Psi(x ; \lambda)=\exp \int \frac{\lambda F^{2}-G H+F H^{\prime}-F^{\prime} H}{G^{2}-u F^{2}-F H+F^{\prime} G-F G^{\prime}} d x,
$$

where prime denotes a derivation in $x$ and

$$
\begin{gathered}
F \equiv-3 u^{\prime \prime \prime}+6 u u^{\prime}-3 c_{1} u^{\prime}+9\left(u-c_{1}\right) \lambda, \quad H \equiv 6\left(u^{\prime \prime}-u^{2}+c_{1} u\right) \lambda-\mu, \\
G \equiv u^{(\mathrm{IV})}+c_{1}\left(u^{\prime \prime}+u^{2}\right)-3 u^{\prime 2}-9 u^{\prime} \lambda-\frac{4}{3} u^{3}-27\left(\lambda^{2}+c_{2}\right), \\
c_{1} \equiv-12 \frac{g_{3}-\widetilde{g}_{3}}{g_{2}-\widetilde{g}_{2}}, \quad c_{2} \equiv \frac{8}{3} \frac{\left(3 \widetilde{g}_{3}+g_{3}\right) g_{2}-\left(3 g_{3}+\widetilde{g}_{3}\right) \widetilde{g}_{2}}{g_{2}-\widetilde{g}_{2}}
\end{gathered}
$$

One particular case of the potential (49) and more general property of finite-gap potentials are discussed in 39.

The natural generalization of Hermite's method is to consider nonlinear homogeneous ansatzs for the $\Psi$-function. For instance, the quadratic ansatz

$$
\Psi=e^{k x} \sum_{j, n} A_{j n} \Phi\left(x-\Omega_{j} ; \alpha\right) \Phi\left(x-\Omega_{n} ; \alpha\right) .
$$

However, this does not fit into the framework of finite-gap integration theory, because the poles of the potential can depend on the spectral parameter. The following example with the transcendental dependence on spectral parameter elucidates this:

$$
\Psi^{\prime \prime}=(6 \wp(x)+2 \wp(x-\lambda)+4 \wp(\lambda)) \Psi, \quad \Psi(x ; \lambda)=\Phi^{2}(x ; \lambda) .
$$


Actually, the quadratic (and higher) ansatzs will not give an advantage due to the relation

$$
\Phi(x ; \alpha)^{2}=-\Phi^{\prime}(x ; 2 \alpha, \zeta(2 \alpha)-2 \zeta(\alpha))
$$

and we again arrive at the framework of Hermite's method.

Note that the nonintegrability of equation (1) in context of the point 3) of the Proposition, nevertheless, can be useful for its integrability with special values of $\lambda$ or for more complex operator pencils with a dependence of the potential (say parameters $a, b, c, d$ in Examples 4-5) on $\lambda$. The availability of additional constants in the potentials may be considered as a family of spectral pencils, and under fixed values of $\lambda$, as new spectral problems. For instance, the 2-gap Lamé potential $u=6 \wp(x)$ for equation (5) is obtained from example (34) with $\lambda=0$ and $d \rightarrow \lambda$, $\Psi^{\prime} \rightarrow \Psi$. A less simple example is to swap the parameters $\lambda \leftrightarrow c$ in equation (36), whereupon one finds the finite-gap operator $\lambda$-pencil

$$
\Psi^{\prime \prime \prime}-12\left(\wp(x)+\lambda^{2}\right) \Psi^{\prime}-(c+12 \lambda \wp(x)) \Psi=0
$$

with the algebraic curve $F(k, \lambda)=0$ of genus $g=8$ [5, formula (15)].

\section{ACKNowledgments}

The author is grateful to Dr. A.Smirnov for helpful discussions. The equation (39) was considered in collaboration with him. The author thanks Prof. R. Conte for a copy of an old paper [7]. My special thanks are due to Profs. E. Previato and V.Enol'skii for much attention to the work and discussions, and Mark van Hoeij for discussions of the computer algorithms. Dr. N. Ustinov made some important observations.

The author also is grateful to Prof. J. C. Eilbeck for numerous discussions, remarks and hospitality at Heriot-Watt University where the paper was improved.

The author was supported by Royal Society/NATO Fellowship and RFBR (0001-00782).

\section{REFERENCES}

1. Belokolos E. D, Bobenko A.I., Enol'skii V.Z., Its A.R., Matveev V.B. Algebro-Geometric Approach to Nonlinear Integrable Equations. Springer-Verlag, Berlin (1994), 337 pp.

2. Belokolos E. D., Enol'skii V.Z. Verdier elliptic solitons and the Weierstrass reduction theory. Func. Anal. and its Appl. (1989), 23(1), 57-58. (in Russian)

3. Brezhnev Yu. V. Darboux transformation and some multi-phase solutions of the DoddBullough-Tzitzeica equation: $U_{x t}=e^{U}-e^{-2 U}$. Phys. Lett. A (1996), 211(2), 94-100.

4. Brezhnev Yu. V. Gröbner bases in the theory of elliptic solitons for the spectral problems of the 3-rd order. Proc. Int. Conf. "Symmetries and Differential Equations". Krasnoyarsk'2000, 53-56. (in Russian)

5. Brezhnev Yu. V. Elliptic Solitons with Free Constants and their Isospectral Deformations. Rep. Math. Phys. (2001), 48(1/2), 39-46.

6. Buchberger B. Gröbner Bases - an Algorithmic Method in Polynomial Ideal Theory. In "Multidimensional Systems Theory" (N. K. Bose, ed.), D. Riedel, Dordrecht (1985).

7. Chazy J. Sur les équations différentielles du troisiéme ordre et d'odre supérieur dont l'intégrable générale a ses points critiques fixes. Thése, Paris (1910). Acta Math. (1911), 34, 317-385.

8. Chudnovsky D. V. Meromorphic solutions of nonlinear PDEs and many-particle completely integrable systems. J. Math. Phys. (1979), 20(12), 2416-2422.

9. Cohen A.M., Cushman R.H. Gröbner Bases and Standard Monomial Theory. Progress in Mathematics (1993), 109, 41-60.

10. Cox D., Little J., O'Shea D. Ideals, Varieties and algorithms (An Introduction to Computational Algebraic Geometry and Commutative Algebra). Springer-Verlag, New York (1992). 
11. Darboux G. Sur une équation linéare. Comptes Rendus Acad. Sci. Paris (1882), XCIV(25), 1645-1648.

12. Dickson R., Gesztesy F., Unterkofler K. Algebro-geometric solutions of the Boussinesq hierarhy. Rev. Math. Phys. (1999), 11(7), 823-879.

13. Dickson R., Gesztesy F., Unterkofler K. A new approach to the Boussinesq hierarchy. Math. Nachr. (1999), 198, 51-108.

14. Eilbeck J. C., Enol'skii V.Z. Elliptic Baker-Akhiezer functions and an application to an integrable dynamical system. J. Math. Phys. (1994), 35(3), 1192-1201.

15. Enolskii V.Z., Eilbeck J.C. On the two-gap locus for the elliptic Calogero-Moser system. J. Phys. A: Math. Gen. (1995), 28, 1069-1088.

16. Enol'skii V.Z., Kostov N. A. On the Geometry of Elliptic Solitons. Acta Appl. Math. (1994), 36, $57-86$.

17. Fordy A., Gibbons J. Some remarkable nonlinear transformations. Phys. Lett. A (1980), $\mathbf{7 5}(5), 325-325$.

18. Forsyth A. R. Theory of Differential Equations. P. I-IV (v. I-VI). Dover Publ., New York (1959).

19. Gavrilov L., Perelomov A.M. On the explicit solutions of the elliptic Calogero system. J. Math. Phys. (1999), 40(12), 6339-6352.

20. Gesztesy F., Weikard R. On Picard potentials. Diff. and Int. Equations (1995), 8(6), 14531476.

21. Gesztesy F., Weikard R. Elliptic algebro-geometric solutions of the KdV and AKNS hierarchies - an analytic approach. Bul. (New Series) Amer. Math. Soc. (1998), 35, 271-317.

22. Gesztesy F., Sticka W. On a theorem of Picard. Proc. Amer. Math. Soc. (1998), 126(4), 10891099.

23. Gesztesy F., Holden H. Hierarchies of Soliton Equations and their Algebro-Geometric Solutions. 1. Cambridge Studies in Advanced Mathematics, Cambridge University Press (2002). (in press)

24. Halphen G. H. Mémoire sur la réduction des équations différentielles linéaires aux formes intégrables. Mem. prés. l'Acad. Sci. France (1884), 28, 1-300.

25. Halphen G. H. Traité des Fonctions Elliptiques et de Leurs Applications. Parte 1. GauthierVillars et Fils., Paris (1886), 492 pp.

26. Halphen G. H. Traité des Fonctions Elliptiques et de Leurs Applications. Parte 2. GauthierVillars et Fils., Paris (1888), 659 pp.

27. Hermite C. Oeuvres de Charles Hermite. III. Gautheir-Villar, Paris (1912).

28. Kamke Dr. E. Differentialgleichungen Lösunsmethoden und Lösungen. New York (1971).

29. Krichever I. M. Elliptic solutions of the Kadomtsev-Petviashvili equation and integrable particle systems. Func. Anal. and its Appl. (1980), 14(4), 45-54. (in Russian)

30. Musette M., Conte R. Bäcklund transformation of the partial differential equations from the Painlevé-Gambier classification. I. Kaup-Kupershmidt equation. J. Math. Phys. (1998), 39(10), 5617-5630.

31. Progress in Mathematics (1993), 109. Computational Algebraic Geometry. (F. Eyssette, A. Galligo, eds.), Birkhäuser.

32. Sawada K., Kotera T. A Method for Finding N-Soliton Solutions of K.d.V. Equation and K.d.V.-Like Equation. Progr. Theor. Phys. (1974), 51(5), 1355-1367.

33. Smirnov A. O. Finite-Gap Elliptic Solutions of the KdV Equation. Acta Appl. Math. (1994), 36, 125-166.

34. Smirnov A. O. Two-gap elliptic solutions of the Boussinesq equation. Mat. Sbornik (1999), 190(5), 139-157. (in Russian)

35. C ${ }^{\text {te }}$ de Sparre. Sur l'équation $\frac{d^{2} y}{d x^{2}}+\left[2 \nu \frac{k^{2} \operatorname{sn} x \operatorname{cn} x}{\operatorname{dn} x}+2 \nu_{1} \frac{\operatorname{sn} x \operatorname{dn} x}{\operatorname{cn} x}-2 \nu_{2} \frac{\operatorname{cn} x \operatorname{dn} x}{\operatorname{sn} x}\right] \frac{d y}{d x}=$ $=\left[\frac{1}{\operatorname{sn}^{2} x}\left(n_{3}-\nu_{2}\right)\left(n_{3}+\nu_{2}+1\right)+\frac{\operatorname{dn}^{2} x}{\operatorname{cn}^{2} x}\left(n_{2}-\nu_{1}\right)\left(n_{2}+\nu_{1}+1\right)+\right.$ $\left.+\frac{k^{2} \operatorname{cn}^{2} x}{\operatorname{dn}^{2} x}\left(n_{1}-\nu\right)\left(n_{1}+\nu+1\right)+k^{2} \operatorname{sn}^{2} x\left(n+\nu+\nu_{1}+\nu_{2}\right)\left(n-\nu-\nu_{1}-\nu_{2}+1\right)+h\right] y$. Acta Math. (1883), 3, 105-140; 289-321.

36. Treibich A. Hyperelliptic tangent covers and finite-gap potentials. Uspekhi Mat. Nauk (2001), 56(6), 89-136. (in Russian).

37. Treibich A., Verdier J. L. Revêtements tangentiels et sommes de 4 nombres triangulaires. Comptes Rendus Acad. Sci. Paris (1990), 311, 51-54. 
38. Unterkofler K. On the solutions of Halphen's equations. Diff. and Int. Equations (2001), 14(9), $1025-1050$.

39. Ustinov N.V., Brezhnev Yu.V. On the $\Psi$-function for the finite-gap potentials. Uspekhi Mat. Nauk (2002), 57(1), 167-168. (in Russian). http://xxx.lanl.gov: hlin.SI/0012039

40. Verdier J. L. New elliptic solitons. Preprint, Paris (1987).

41. Wang D. M. Characteristic Sets and Zero Structure of Polynomial Sets. Lecture Notes, RISCLINZ, Johannes Kepler University, Austria, (1989).

42. Wang D. An Elimination Method Based on Seidenberg's Theory and its Applications. Progress in Mathematics (1993), 109, 301-328.

Theoretical Physics Department, Kaliningrad State University, A. Nevsky st. 14, KaLiningRAD 236041, RUSSiA.

E-mail address: brezhnev@mail.ru

Current address: Department of Mathematics, Heriot-Watt University, Edinburgh EH14 4AS, United Kingdom.

E-mail address: brezhnev@ma.hw.ac.uk 